\title{
Bronchoalveolar Lavage Fluid
}

National Cancer Institute

\section{Source}

National Cancer Institute. Bronchoalveolar Lavage Fluid. NCI Thesaurus. Code C13195.

Fluid introduced into, and collected from, the lungs by a bronchoalveolar lavage procedure. 